\title{
Optical Coherence Tomography as a Tool for Ocular Dynamics Estimation
}

\author{
Damian Siedlecki, Waldemar Kowalik, and Henryk Kasprzak \\ Department of Optics and Photonics, Wroclaw University of Technology, Wybrzeze Wyspianskiego 27, 50-370 Wroclaw, Poland \\ Correspondence should be addressed to Damian Siedlecki; damian.siedlecki@pwr.edu.pl
}

Received 28 February 2015; Revised 4 June 2015; Accepted 11 June 2015

Academic Editor: Ki H. Park

Copyright ( 2015 Damian Siedlecki et al. This is an open access article distributed under the Creative Commons Attribution License, which permits unrestricted use, distribution, and reproduction in any medium, provided the original work is properly cited.

\begin{abstract}
Purpose. The aim of the study is to demonstrate that the ocular dynamics of the anterior chamber of the eye can be estimated quantitatively by means of optical coherence tomography (OCT). Methods. A commercial high speed, high resolution optical coherence tomographer was used. The sequences of tomographic images of the iridocorneal angle of three subjects were captured and each image from the sequence was processed in MATLAB environment in order to detect and identify the contours of the cornea and iris. The data on pulsatile displacements of the cornea and iris and the changes of the depth of the gap between them were retrieved from the sequences. Finally, the spectral analysis of the changes of these parameters was performed. Results. The results of the temporal and spectral analysis manifest the ocular microfluctuation that might be associated with breathing (manifested by $0.25 \mathrm{~Hz}$ peak in the power spectra), heart rate (1-1.5 Hz peak), and ocular hemodynamics $(3.75-4.5 \mathrm{~Hz}$ peak). Conclusions. This paper shows that the optical coherence tomography can be used as a tool for noninvasive estimation of the ocular dynamics of the anterior segment of the eye, but its usability in diagnostics of the ocular hemodynamics needs further investigations.
\end{abstract}

\section{Introduction}

For over 20 years, optical coherence tomography has evolved from an emerging imaging technology of uncertain future to a well-established diagnostic tool being used in many different fields of medicine. Due to its unique features of visualizing transparent and semitransparent objects with an ultrahigh resolution, it has found the greatest interest in ophthalmology, particularly in imaging of the fine structures of the cornea $[1,2]$ and the retina [3-5]. Furthermore, a significant development of the instrumentation and data processing techniques [6-8] has opened OCT to brand new applications in ocular imaging, measurement, and diagnostics, hardly achievable with other technologies.

Ocular dynamics is a complex phenomenon which is related to the hemodynamics (pulse) [9-12] and mechanical parameters of the eye globe [13-20]. It has been shown before that the rapid variations of the intraocular pressure (IOP), known as ocular pulse, are manifested by the axial and radial displacements of the corneal surface [9] as the most external and the easiest to access structure of the eye globe. Also the simultaneous micromovements of the retina were observed as a result of the ocular pulsation [21-23]. Microfluctuation of the iris and/or crystalline lens that might be affected by the intraocular pressure variation related to the retinal blood flow was reported in other studies, as well [24].

In the existing literature, the term "dynamics" used in relation to the iridocorneal angle is usually associated either with its changes with light-dark adaptation $[25,26]$ or drug application [27], and in both of these cases the tomographic images of the angles are captured as a single shot before and after changing the conditions of the experiment. The aim of the current study is to present a new approach to the dynamic changes of the anterior segment of the eye. In this approach it can be continuously examined in a short period of time, with a relatively high frequency (of more than $20 \mathrm{~Hz}$ ). The short-term microfluctuation of the anterior chamber angle can be successfully visualized by means of a high speed spectral domain OCT (SD-OCT). The current paper presents also a preliminary example of the analysis of these pulsatile fluctuations, including their spectral analysis, related to the breathing and blood pulsation. 


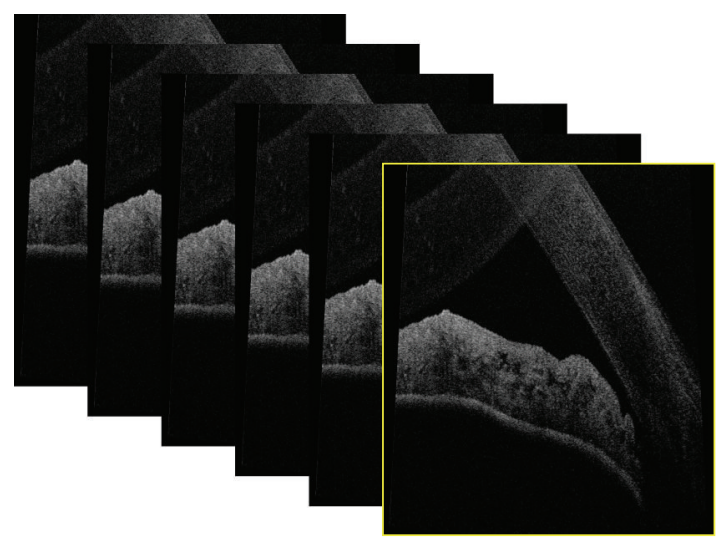

Sequence of original images

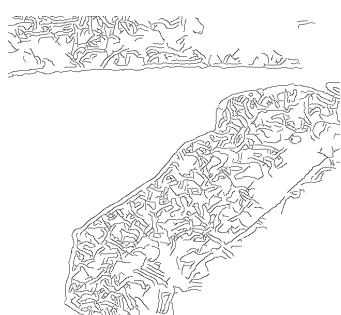

Edge detection

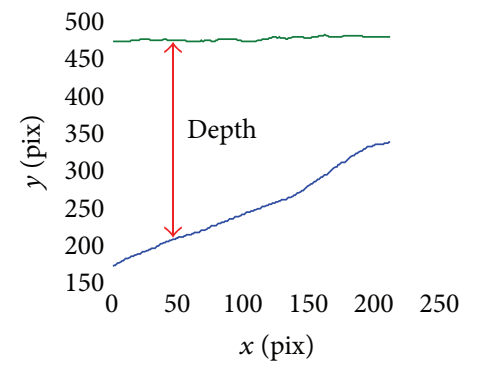

- Anterior iris

_ Posterior cornea

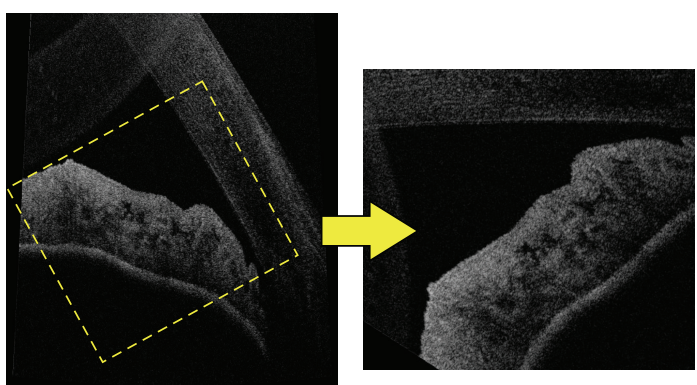

Image crop and rotation

Posterior cornea and anterior iris identification
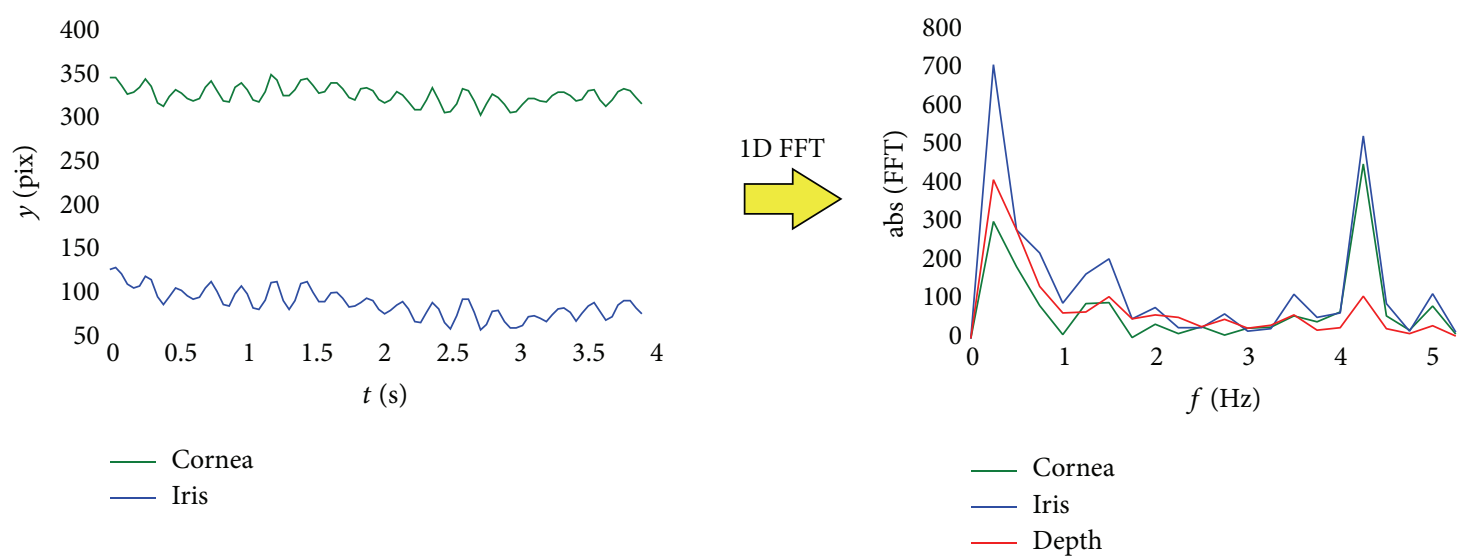

Temporal and spectral analysis

FIGURE 1: The stages of the image processing for the reconstruction of the posterior surface of the cornea and the anterior surface of the iris. The effect of the "bended" cornea on the original image is an artefact that can be associated with the Fourier transform of the optical signal reaching the light detector and the relatively short axial range of the instrument (c.a. $2.5 \mathrm{~mm}$ ). The image of superposition of the identified surfaces and data after edge detection is only for demonstration purposes.

\section{Methods}

2.1. In Vivo Measurements. Three patients, aged from 65 to 70 , participated in the study. Subject \#1 was diagnosed with angle closure glaucoma (ACG) and Subjects \#2 and \#3 were diagnosed with open-angle glaucoma (OAG). The iridocorneal angle in the temporal quadrant of their eyes (OS for Subject \#1, OD for Subjects \#2 and \#3) was imaged with use of a SD-OCT Copernicus HR (Optopol Technology,
Zawiercie, Poland). It is a high axial resolution (up to $3 \mu \mathrm{m}$ in tissue), high speed (up to $52000 \mathrm{~A}$-scans/sec, according to the device specifications) spectral domain OCT, using a $850 \mathrm{~nm}$ superluminescent diode (SLED) as a light source. It is optionally equipped with an attachable anterior segment adapter, which makes it useful for the anterior chamber measurement purposes. The standard examination mode denoted as "anterior" and the scanning protocol denoted as "animation" were used. This particular protocol enables 

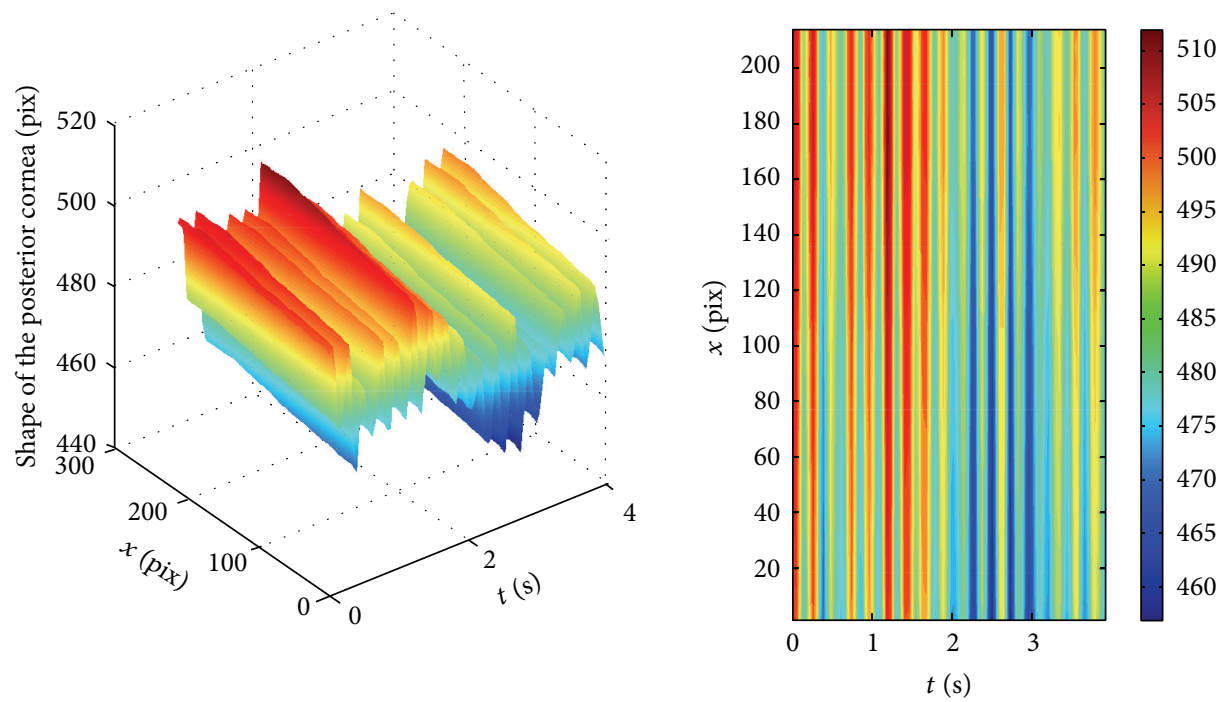

(a)
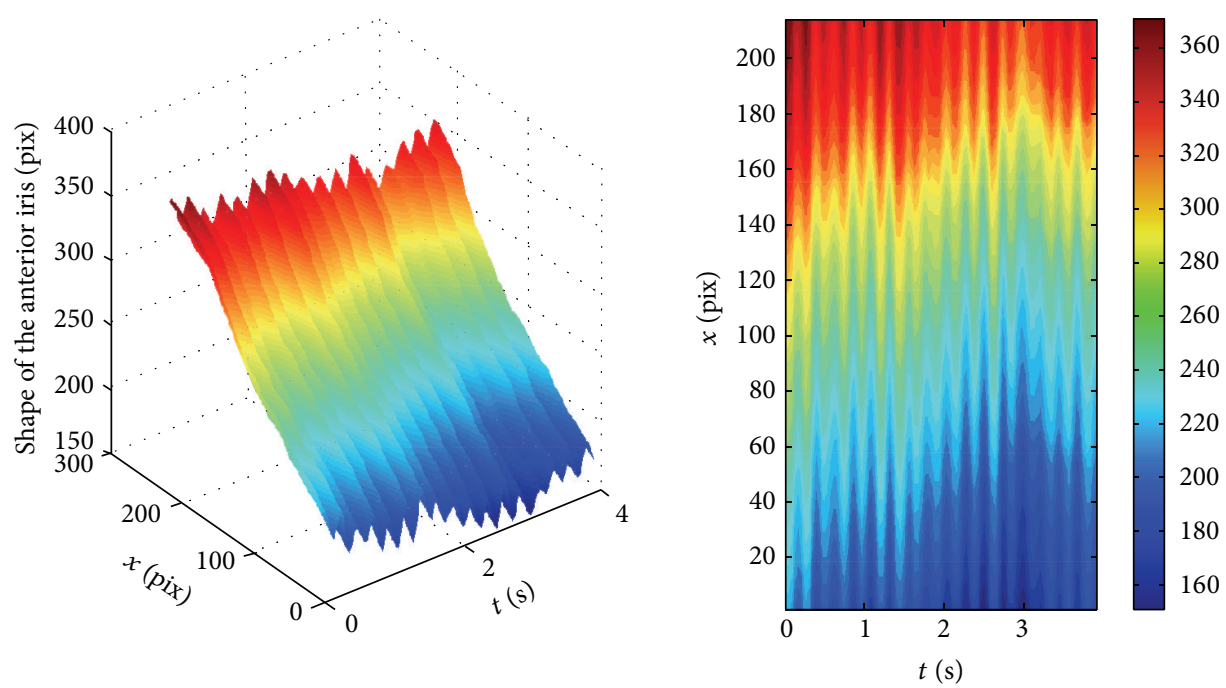

(b)
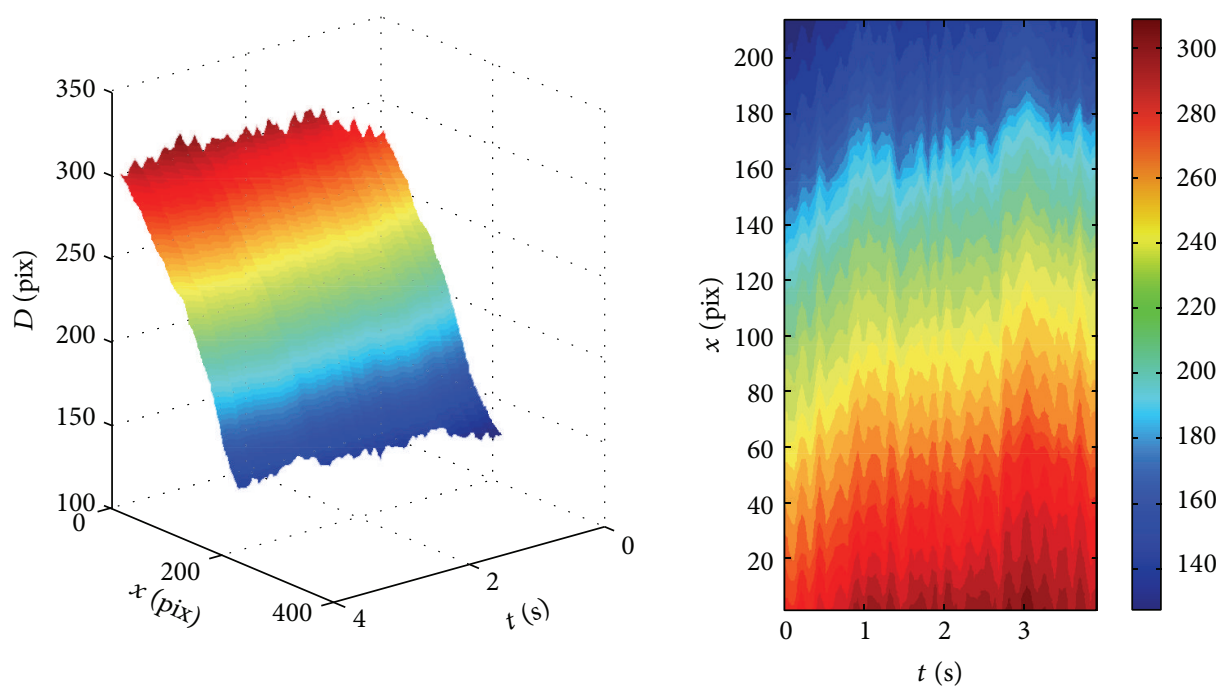

(c)

FIgURE 2: An example of temporal fluctuation of the (a) posterior cornea, (b) anterior iris, and (c) depth $D$ of the gap between the cornea and the iris being a result of the data processing of the sequence captured for Subject \#1. Both plots in the row represent the same data, but the pulsations are better demonstrated in the contour plots. 


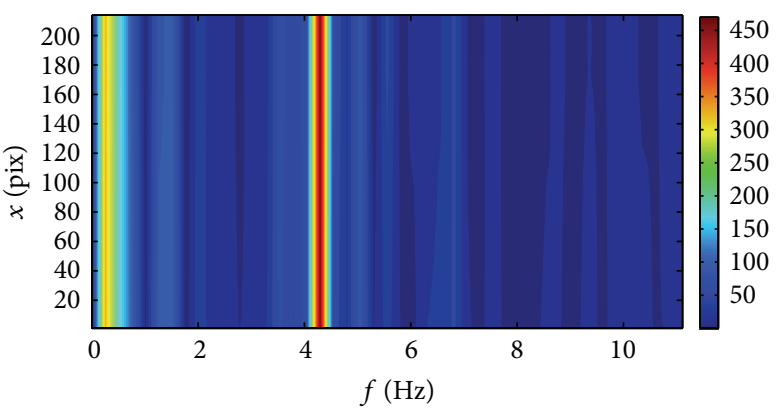

(a)

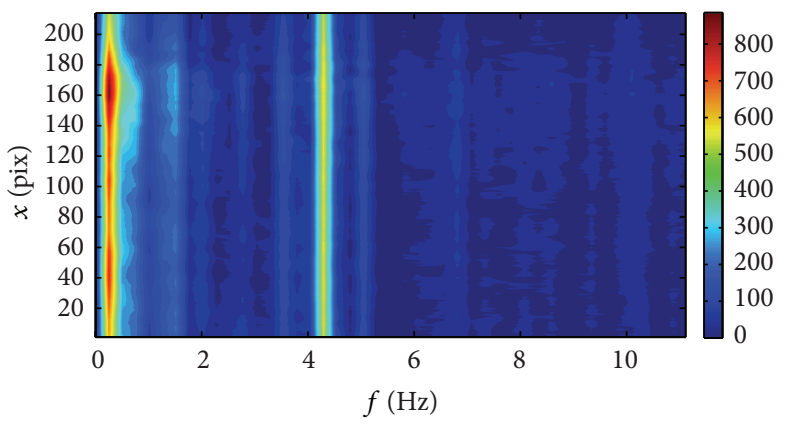

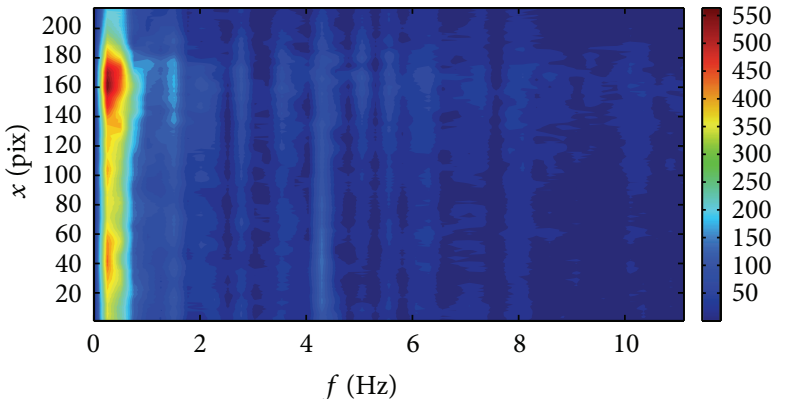

(b)

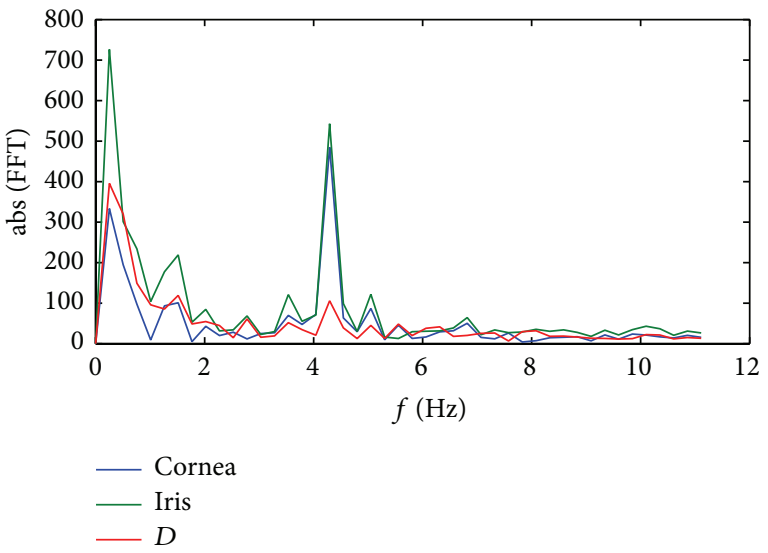

(d)

FIGURE 3: Fourier spectra of displacement data obtained from the sequence captured for Subject \#1: (a) the results for the posterior surface of the cornea; (b) the results for the anterior surface of the iris; (c) the results for the distance $D$ between the cornea and iris; (d) the power spectra averaged over the $x$ coordinate. The spectral analysis was performed as the one-dimensional fast Fourier transform of the temporal changes of the surfaces.

capturing the sequence of cross-sectional images (B-scans) in the same meridional position. The length of time of the sequence depends on the lateral density of a single image (the number of A-scans contributed to a single Bscan). The maximum number of A-scans in this scanning protocol is 1800 . This enables the capturing of the sequence of $3.96 \mathrm{~s}$ long (as estimated by the standard software distributed together with the device), containing a series of 90 images of the iridocorneal angle, in the same meridional crosssection. This configuration was used for the purposes of this study.

During the measurements the subject was fixating centrally on the internal target and his head was stabilized in the chin- and head-rest of the device. He was allowed to breathe freely and was asked not to blink during the sequence acquisition.

All patients provided written informed consent before enrolling in the study. The measurement protocol had been approved by the Institutional Review Board and met the tenets of the Declaration of Helsinki.

2.2. Data Processing. The results of the measurement for each patient contained a series of 90 consecutive cross-sectional images of a resolution $900 \times 1009$ pixels, captured with the frequency of $22.7 \mathrm{~Hz}$ (90 frames captured within $3.96 \mathrm{~s}$ ). Each image was processed in MATLAB with use of a customdeveloped script. The semiautomatic procedures included (a) the selection of the area of interest and its rotation so that the cornea was situated in the upper part of the image; (b) the edge detection [28]; and (c) the identification of the posterior surface of the cornea and the anterior surface of the iris. Because of the different scattering properties of the cornea and iris (the cornea has lower signal-to-noise ratio than the iris), several existing algorithms [29-31] of edge and contour detection needed to be tested with various parameters, before the results presented in the next section were obtained. The horizontal range, in which the surfaces of the cornea and iris could be unerringly identified automatically, was selected subjectively by the software operator. The coordinates of the start and end points of this range depended on the observed movability of the ocular structures in the whole sequence. The stages of the image processing used in the study are presented in Figure 1.

After identification of the posterior cornea and anterior iris surfaces on each particular scan from the sequence, it was possible to show variability of their temporal position. Their observed displacements might be associated with the fast, dynamic changes of either the internal conditions of 


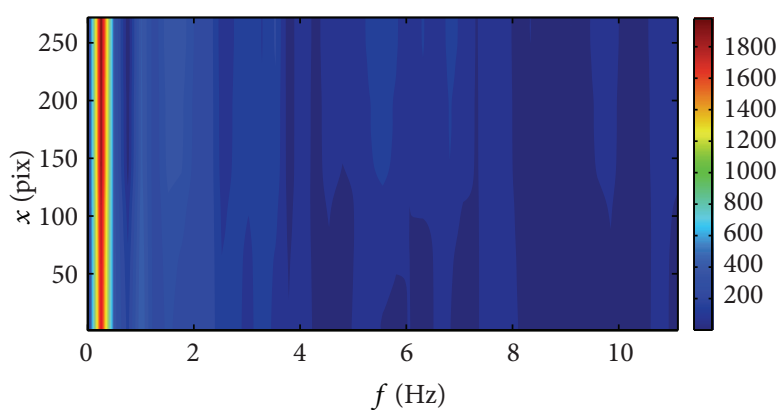

(a)

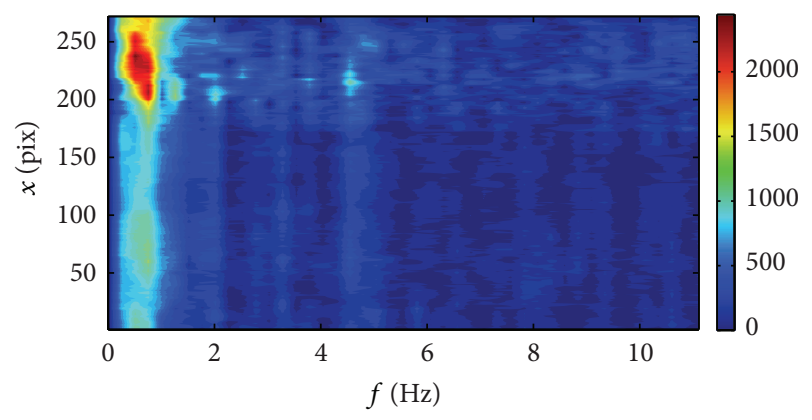

(c)

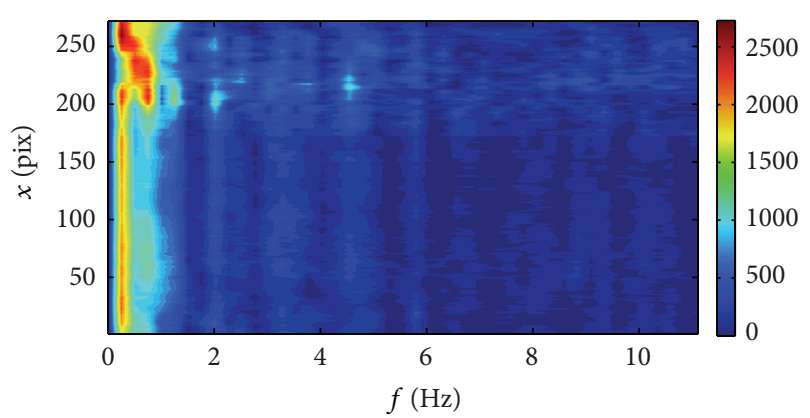

(b)

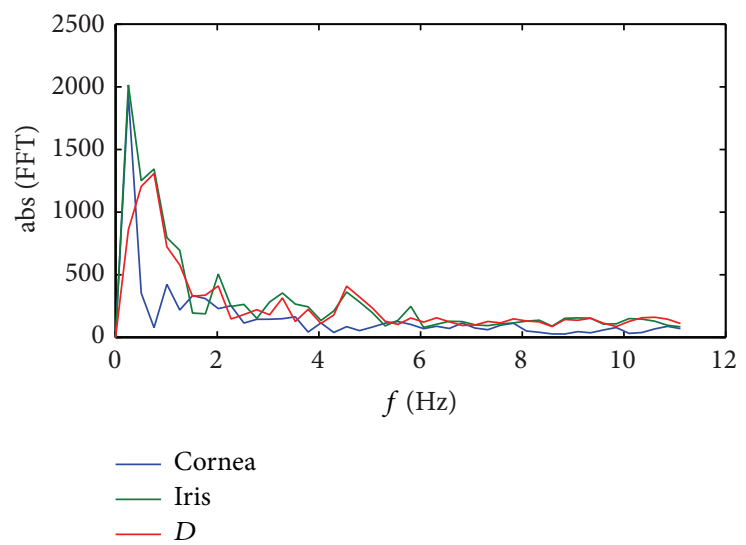

(d)

FIGURE 4: Fourier spectra of displacement data obtained from the sequence captured for Subject \#2: (a) the results for the posterior surface of the cornea; (b) the results for the anterior surface of the iris; (c) the results for the distance $D$ between the cornea and iris; (d) the power spectra averaged over the $x$ coordinate. The spectral analysis was performed as the one-dimensional fast Fourier transform of the temporal changes of the surfaces.

the eye globe, such as ocular pulse and muscle contraction/relaxation, or external ones (i.e., breathing). For this purpose, the depth $D$ of the gap between the posterior surface of the cornea and anterior surface of the iris along each pixel column in the rotated image was calculated in terms of pixels. The spectral analysis of the variability of the position in time was performed as well, as one-dimensional fast Fourier transform (FFT) of the temporal changes of the location of the identified structures (cornea and iris and its longitudinal separation) in each particular pixel column. As a result, the spectral map of the fluctuations in position of each pixel was plotted.

\section{Results}

The sequences showing the dynamic fluctuations of the iridocorneal region captured for all the subjects participating in the study can be seen on the videos (see Video, Supplemental Digital Content 1, captured for Subject \#1; see Video, Supplemental Digital Content 2, captured for Subject \#2; see Video, Supplemental Digital Content 3, captured for Subject \#3, in Supplementary Material available online at http://dx.doi.org/10.1155/2015/293693). The frame per second (fps) rate of the videos is two times lower than the acquisition rate of the OCT instrument.
Figure 2 presents the microfluctuation of the cornea and iris captured for Subject \#1. At first glance, the same behavior can be observed for all the subjects participating in the study. The depth $D$ between the posterior surface of the cornea and the anterior surface of the iris undergoes some small changes, as well, which indicates small difference between the amplitudes of the pulsatile movements of the cornea and the iris. However, the spectral analysis shows slightly different characteristics of these movements for each patient, which can be seen in Figures 3-5.

It needs to be emphasized that the results presented here are not the results of the measurements of the iridocorneal angle itself, which are expressed in terms of angles. The results presented above are rather the results of the measurements of the distance between the posterior cornea and anterior surface of the iris, expressed in pixels. Of course, they are associated with the iridocorneal angle; however, the tomographic images were not corrected from the optical distortion, and instead of using the units of angles and micrometers, it was more appropriate to use pixels.

\section{Discussion}

There are three basic features that are manifested in all the spectral plots for all the subjects. The first one is the peak 


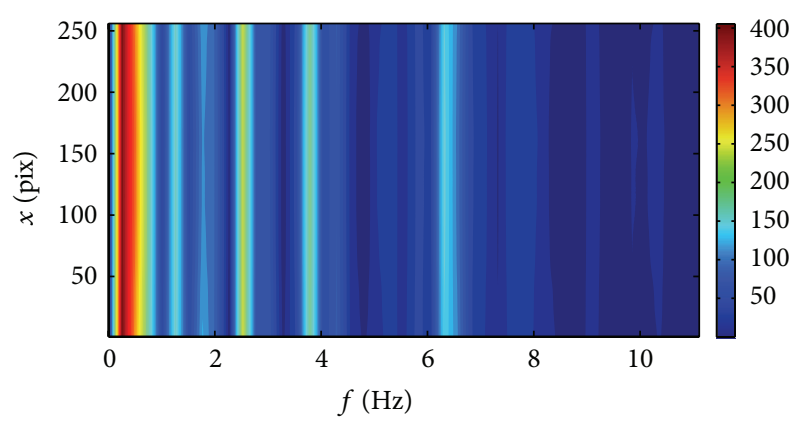

(a)

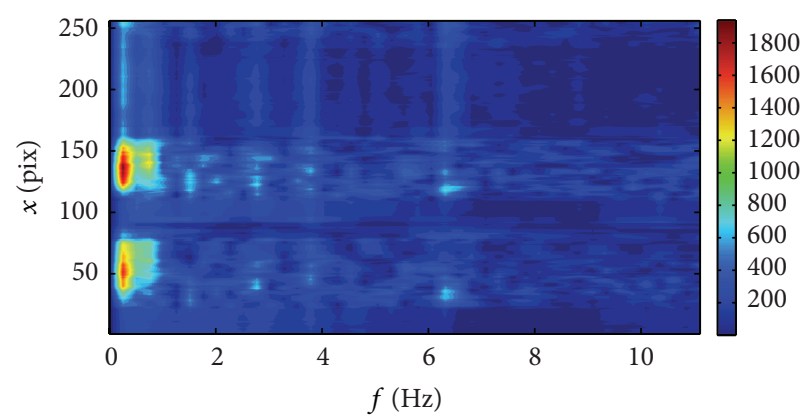

(c)

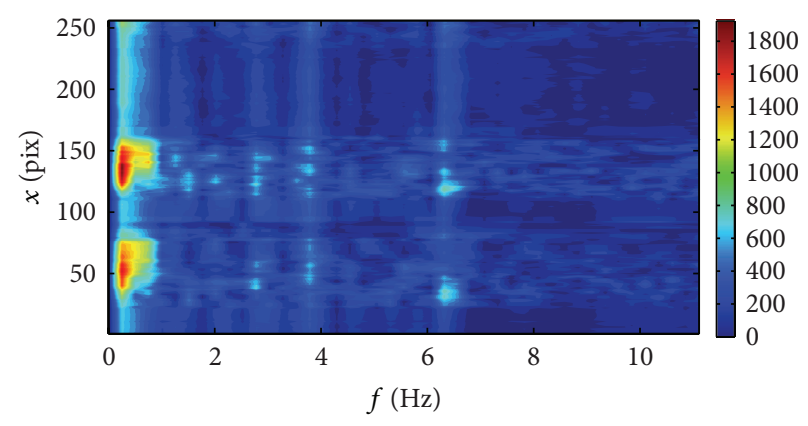

(b)

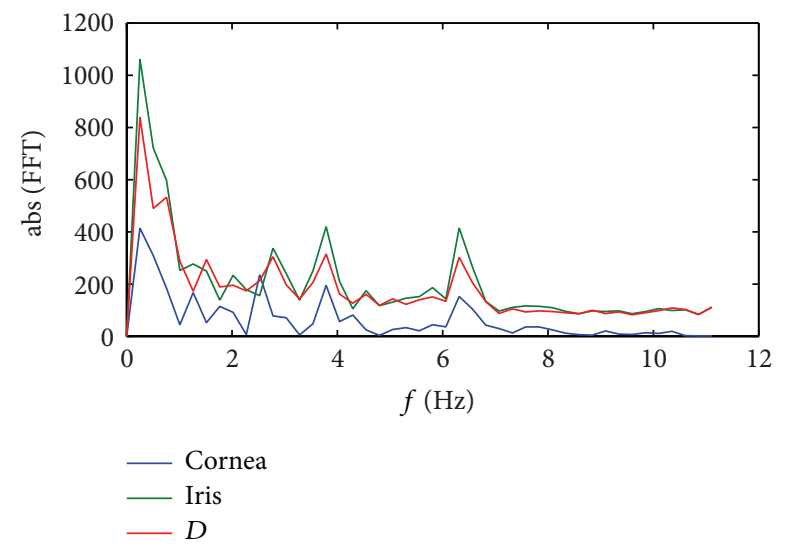

(d)

FiguRE 5: Fourier spectra of displacement data obtained from the sequence captured for Subject \#3: (a) the results for the posterior surface of the cornea; (b) the results for the anterior surface of the iris; (c) the results for the distance $D$ between the cornea and iris; (d) the power spectra averaged over the $x$ coordinate. The spectral analysis was performed as the one-dimensional fast Fourier transform of the temporal changes of the surface.

for the low frequency (about $0.25 \mathrm{~Hz}$ ) that is likely associated with breathing $[32,33]$ and respective head movement during measurement [34].

The second feature is the small peak that appears in the frequency range between 1 and $1.5 \mathrm{~Hz}$. These frequencies can be linked with blood pulsation in the ocular vessels $[32,33]$. An interesting fact is that for Subject \#1 this peak is quite distinguishable for both the posterior cornea and anterior iris and the distance $D$ between them. For Subject \#3 this peak is visible for cornea and iris, but not for the depth $D$, whereas for Subject \#2 this peak moves slightly from about $1 \mathrm{~Hz}$ for the cornea to about $0.75 \mathrm{~Hz}$ for the iris and the distance $D$, and for the $D$ its magnitude is even higher than the peak associated with breathing. It can be the effect of the extensive peak of a large amplitude, which appears for pixel numbers larger than 200. This is the region of a step-like structure on the surface of the iris. Its lateral movements are manifested as changes of a very large amplitude in depth. This peak seems to cover (overlap) the neighboring frequencies which results in a frequency drift in the averaged spectrum for iris and depth noticeable in Figure 4(a).

The third common feature is the local, well distinct peak, with the maximum for the frequencies varying from $3.75 \mathrm{~Hz}$ (for Subject \#3) to $4.5 \mathrm{~Hz}$ (for Subject \#2). These peaks can be possibly related to the harmonics of the blood pulsation. For example, the results for Subject \#3 (Figure 5) present the local peaks at frequencies $1.25,2.5,3.75$, and $6.25 \mathrm{~Hz}$, which are multiples of $1.25 \mathrm{~Hz}$. For other subjects these harmonics are not so obvious, probably due to low sampling rate in the frequency domain or due to nonstationarity of the signal. In order to improve this analysis one should use OCT with possibility of recording longer sequences. As shown in the paper [34] the involuntary head movement can also influence the appearance of higher harmonics in the cornea position.

Some recent studies reported the phenomenon of ocular pulse dicrotism $[35,36]$, which is related to the condition (rigidity) of the arterial vessels and can be observed in older subjects. Since all the subjects in the current study are aged from 65 to 70, it is likely that the ocular pulse dicrotism could also be related to our observations. Further studies should give an answer, where explanation is more probable.

The number of the peaks that can be clearly and uniquely distinguished in the spectral characteristics of the movement of the anterior segment of the eye is different for various subjects. The origin of the maxima other than the one described above is not clear yet and can be the subject of another study.

\section{Conclusions}

This paper is a preliminary study of ocular dynamics, its imaging, and analysis. To the best of the authors' knowledge, 
this paper is the very first demonstration of the rapid dynamic fluctuations of the geometry of the iridocorneal part of the eye. The imaging was performed with use of a commercially available instrument with high acquisition rate.

The power spectra of the pulsatile micromovements of the whole anterior segment can provide some information about individual parameters of a subject eye. These parameters are the mechanical response of the ocular structures on the pulsation in the vitreous body and may undergo some discrete changes with the progress of the pathological states. However, the potential of the method as a tool in early diagnosis of the ocular diseases, in particular glaucoma, is still to be studied.

One of the problems that has to be overcome for the further development of the method is the stabilization of the images from the sequence of the tomograms, because some undesired lateral movements of the eye can be seen on the movies presented in videos. The significance of these lateral movements on the overall spectral characteristics was not the subject of the current study, and it was not estimated. However, this problem can be solved numerically, when the stabilization of each particular tomogram is performed. The development of a fully automatic image segmentation and surface identification [37-39] would also be helpful.

Further research on larger population samples with simultaneous use of other diagnostic techniques (i.e., pulse oximetry) would give an answer to the question of whether the presented method of ocular dynamics estimation by means of optical coherence tomography might be used for the diagnostics of ocular disorders associated with hemodynamics of the eye, such as glaucoma.

\section{Disclosure}

Part of this work was presented in poster form at the 7th European and 1st World Meeting on Visual and Physiological Optics, 25-27 August 2014, Wroclaw, Poland.

\section{Conflict of Interests}

The authors declare that there is no conflict of interests regarding the publication of this paper.

\section{Acknowledgment}

This work was supported by the Polish National Centre of Research under Grant NR13-0012-10/2010.

\section{References}

[1] T. Schmoll, A. Unterhuber, C. Kolbitsch, T. Le, A. Stingl, and R. Leitgeb, "Precise thickness measurements of Bowman's layer, epithelium, and tear film," Optometry and Vision Science, vol. 89, no. 5, pp. E795-E802, 2012.

[2] R. Yadav, R. Kottaiyan, K. Ahmad, and G. Yoon, "Epithelium and Bowman's layer thickness and light scatter in keratoconic cornea evaluated using ultrahigh resolution optical coherence tomography," Journal of Biomedical Optics, vol. 17, no. 11, Article ID 116010, 2012.
[3] W. Drexler, U. Morgner, R. K. Ghanta, F. X. Kärtner, J. S. Schuman, and J. G. Fujimoto, "Ultrahigh-resolution ophthalmic optical coherence tomography," Nature Medicine, vol. 7, no. 4, pp. 502-506, 2001.

[4] D. Cabrera Fernández, H. M. Salinas, and C. A. Puliafito, "Automated detection of retinal layer structures on optical coherence tomography images," Optics Express, vol. 13, no. 25, pp. 10200-10216, 2005.

[5] A. Mishra, A. Wong, K. Bizheva, and D. A. Clausi, "Intra-retinal layer segmentation in optical coherence tomography images," Optics Express, vol. 17, no. 26, pp. 23719-23728, 2009.

[6] A. de Castro, S. Ortiz, E. Gambra, D. Siedlecki, and S. Marcos, "Three-dimensional reconstruction of the crystalline lens gradient index distribution from OCT imaging," Optics Express, vol. 18, no. 21, pp. 21905-21917, 2010.

[7] D. Siedlecki, A. de Castro, E. Gambra et al., "Distortion correction of OCT images of the crystalline lens: gradient index approach," Optometry and Vision Science, vol. 89, no. 5, pp. E709-E718, 2012.

[8] S. Ortiz, P. Pérez-Merino, S. Durán et al., "Full OCT anterior segment biometry: an application in cataract surgery," Biomedical Optics Express, vol. 4, no. 3, pp. 387-396, 2013.

[9] R. B. Northrop and S. S. Nilakhe, "A no touch ocular pulse measurement system for the diagnosis of carotid occlusions," IEEE Transactions on Biomedical Engineering, vol. 24, no. 2, pp. 139-148, 1977.

[10] M. E. Langham, R. A. Farrell, V. O’Brien, D. M. Silver, and P. Schilder, "Blood flow in the human eye," Acta Ophthalmologica. Supplement, vol. 191, pp. 9-13, 1989.

[11] D. R. Trew, C. B. James, S. H. L. Thomas, R. Sutton, and S. E. Smith, "Factors influencing the ocular pulse-the heart rate," Graefe's Archive for Clinical and Experimental Ophthalmology, vol. 229, no. 6, pp. 553-556, 1991.

[12] T. M. Bosley, M. S. Cohen, W. Gee, J. Reed III, R. C. Sergott, and P. J. Savino, "Amplitude of the ocular pneumoplethysmography waveform is correlated with cardiac output," Stroke, vol. 24, no. 1, pp. 6-9, 1993.

[13] R. Kempf, Y. Kurita, Y. Iida et al., "Dynamic properties of human eyes," in Proceedings of the 27th Annual International Conference of the Engineering in Medicine and Biology Society (EMBS '05), pp. 3180-3183, Shanghai, China, September 2005.

[14] J. Liu and C. J. Roberts, "Influence of corneal biomechanical properties on intraocular pressure measurement: quantitative analysis," Journal of Cataract and Refractive Surgery, vol. 31, no. 1, pp. 146-155, 2005.

[15] H. A. Weeber, G. Eckert, F. Soergel, C. H. Meyer, W. Pechhold, and R. G. L. van der Heijde, "Dynamic mechanical properties of human lenses," Experimental Eye Research, vol. 80, no. 3, pp. 425-434, 2005.

[16] M. Asejczyk-Widlicka, R. A. Schachar, and B. K. Pierscionek, "Optical coherence tomography measurements of the fresh porcine eye and response of the outer coats of the eye to volume increase," Journal of Biomedical Optics, vol. 13, no. 2, Article ID 024002, 2008.

[17] M. Asejczyk-Widlicka, W. Śródka, R. A. Schachar, and B. K. Pierścionek, "Material properties of the cornea and sclera: a modelling approach to test experimental analysis," Journal of Biomechanics, vol. 44, no. 3, pp. 543-546, 2011.

[18] M. A. Kowalska, H. T. Kasprzak, D. R. Iskander, M. Danielewska, and D. Mas, "Ultrasonic in vivo measurement of ocular surface expansion," IEEE Transactions on Biomedical Engineering, vol. 58, no. 3, pp. 674-680, 2011. 
[19] B. Coudrillier, J. Tian, S. Alexander, K. M. Myers, H. A. Quigley, and T. D. Nguyen, "Biomechanics of the human posterior sclera: Age- and glaucoma-related changes measured using inflation testing," Investigative Ophthalmology and Visual Science, vol. 53, no. 4, pp. 1714-1728, 2012.

[20] M. E. Danielewska, D. R. Iskander, M. A. Kowalska, and H. T. Kasprzak, "Phase dependencies between longitudinal corneal apex displacement and cardiovascular signals: is the ocular pulse influenced by the electrical activity of the heart?" Clinical and Experimental Optometry, vol. 95, no. 6, pp. 631-637, 2012.

[21] K. Singh, C. Dion, S. Costantino, M. Wajszilber, M. R. Lesk, and T. Ozaki, "Development of a novel instrument to measure the pulsatile movement of ocular tissues," Experimental Eye Research, vol. 91, no. 1, pp. 63-68, 2010.

[22] K. Singh, C. Dion, M. Wajszilber, T. Ozaki, M. R. Lesk, and S. Costantino, "Measurement of ocular fundus pulsation in healthy subjects using a novel Fourier-domain optical coherence tomography," Investigative Ophthalmology and Visual Science, vol. 52, no. 12, pp. 8927-8932, 2011.

[23] C. Dion, K. Singh, T. Ozaki, M. R. Lesk, and S. Costantino, "Analysis of pulsatile retinal movements by spectral-domain low-coherence interferometry: influence of age and glaucoma on the pulse wave," PLoS ONE, vol. 8, no. 1, Article ID e54207, 2013.

[24] W. Nowak, A. Hachoł, and H. Kasprzak, "Time-frequency analysis of spontaneous fluctuation of the pupil size of the human eye," Optica Applicata, vol. 38, no. 2, pp. 469-480, 2008.

[25] C. K. S. Leung, W.-M. Chan, C. Y. Ko et al., "Visualization of anterior chamber angle dynamics using optical coherence tomography," Ophthalmology, vol. 112, no. 6, pp. 980-984, 2005.

[26] C. K.-S. Leung, C. Y. L. Cheung, H. Li et al., "Dynamic analysis of dark-light changes of the anterior chamber angle with anterior segment OCT,' Investigative Ophthalmology and Visual Science, vol. 48, no. 9, pp. 4116-4122, 2007.

[27] J. M. Kim, K. H. Park, S. Y. Han et al., "Changes in intraocular pressure after pharmacologic pupil dilation," BMC Ophthalmology, vol. 12, no. 1, article 53, 2012.

[28] G. Azzopardi and N. Petkov, "A CORF computational model of a simple cell that relies on LGN input outperforms the Gabor function model," Biological Cybernetics, vol. 106, no. 3, pp. 177$189,2012$.

[29] J. Malik, S. Belongie, T. Leung, and J. Shi, "Contour and texture analysis for image segmentation," International Journal of Computer Vision, vol. 43, no. 1, pp. 7-27, 2001.

[30] J. Canny, "A computational approach to edge detection," IEEE Transactions on Pattern Analysis and Machine Intelligence, vol. 8, no. 6, pp. 679-698, 1986.

[31] Y. T. Kong and A. Rosenfeld, Topological Algorithms for Digital Image Processing, Elsevier Science, New York, NY, USA, 1996.

[32] M. Collins, B. Davis, and J. Wood, "Microfluctuations of steadystate accommodation and the cardiopulmonary system," Vision Research, vol. 35, no. 17, pp. 2491-2502, 1995.

[33] M. Zhu, M. J. Collins, and D. R. Iskander, "Microfluctuations of wavefront aberrations of the eye," Ophthalmic and Physiological Optics, vol. 24, no. 6, pp. 562-571, 2004.

[34] H. T. Kasprzak and D. R. Iskander, "Ultrasonic measurement of fine head movements in a standard ophthalmic headrest," IEEE Transactions on Instrumentation and Measurement, vol. 59, no. 1, pp. 164-170, 2010.

[35] M. E. Danielewska, D. R. Iskander, and P. KrzyzanowskaBerkowska, "Age-related changes in corneal pulsation: ocular dicrotism," Optometry and Vision Science, vol. 91, no. 1, pp. 5459, 2014.

[36] M. E. Danielewska, P. Krzyżanowska-Berkowska, and D. R. Iskander, "Glaucomatous and age-related changes in corneal pulsation shape. The ocular dicrotism," PLoS ONE, vol. 9, no. 7, Article ID e102814, 2014.

[37] R. Koprowski and Z. Wrobel, "The cell structures segmentation," in Computer Recognition Systems: Proceedings of the 4th International Conference on Computer Recognition Systems CORES '05, M. Kurzyński, E. Puchała, M. Woźniak, and A. Żołnierek, Eds., vol. 30 of Advances in Soft Computing, pp. 569576, Springer, Berlin, Germany, 2005.

[38] R. Koprowski, S. J. Teper, B. Weglarz, E. Wylegała, M. Krejca, and Z. Wróbel, "Fully automatic algorithm for the analysis of vessels in the angiographic image of the eye fundus," BioMedical Engineering Online, vol. 11, article 35, 2012.

[39] R. Koprowski, Z. Wróbel, S. Wilczyński, A. Nowińska, and E. Wylęgała, "Methods of measuring the iridocorneal angle in tomographic images of the anterior segment of the eye," BioMedical Engineering Online, vol. 12, article 40, 16 pages, 2013. 


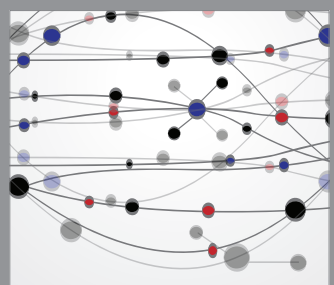

The Scientific World Journal
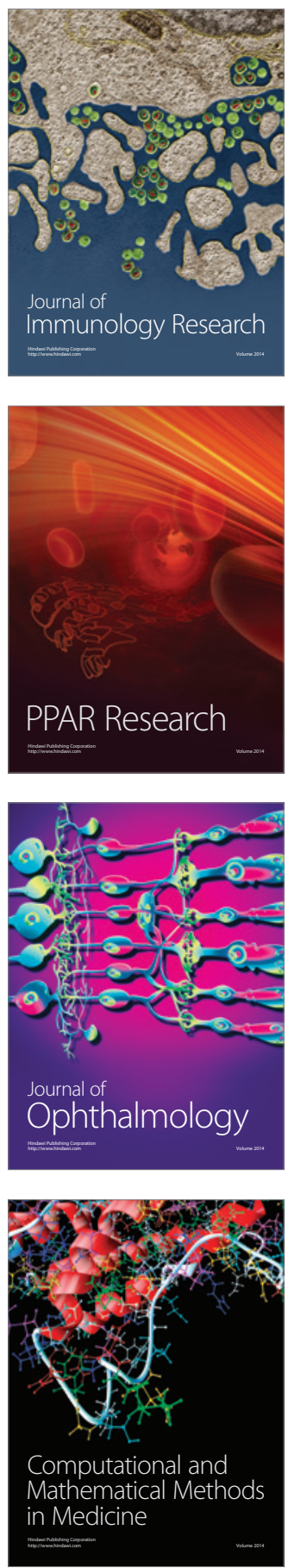

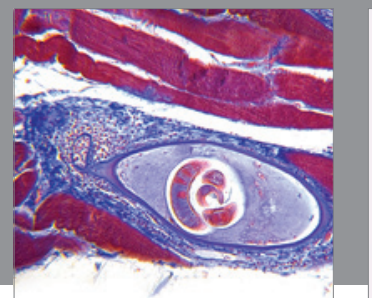

Gastroenterology

Research and Practice
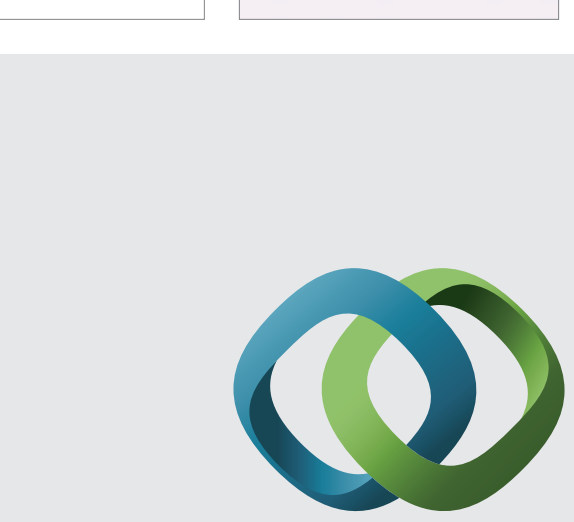

\section{Hindawi}

Submit your manuscripts at

http://www.hindawi.com
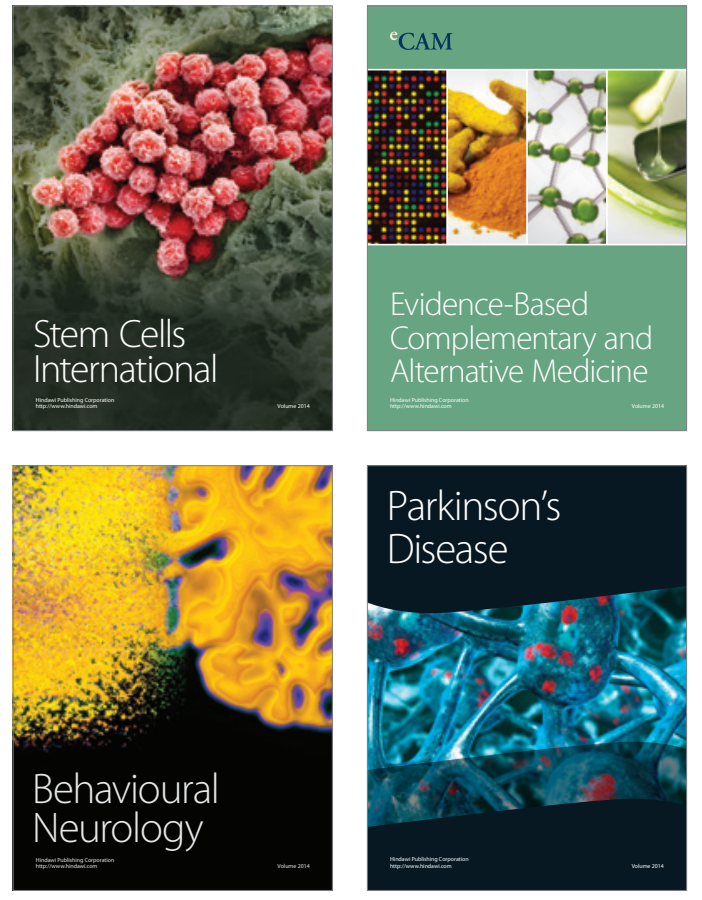
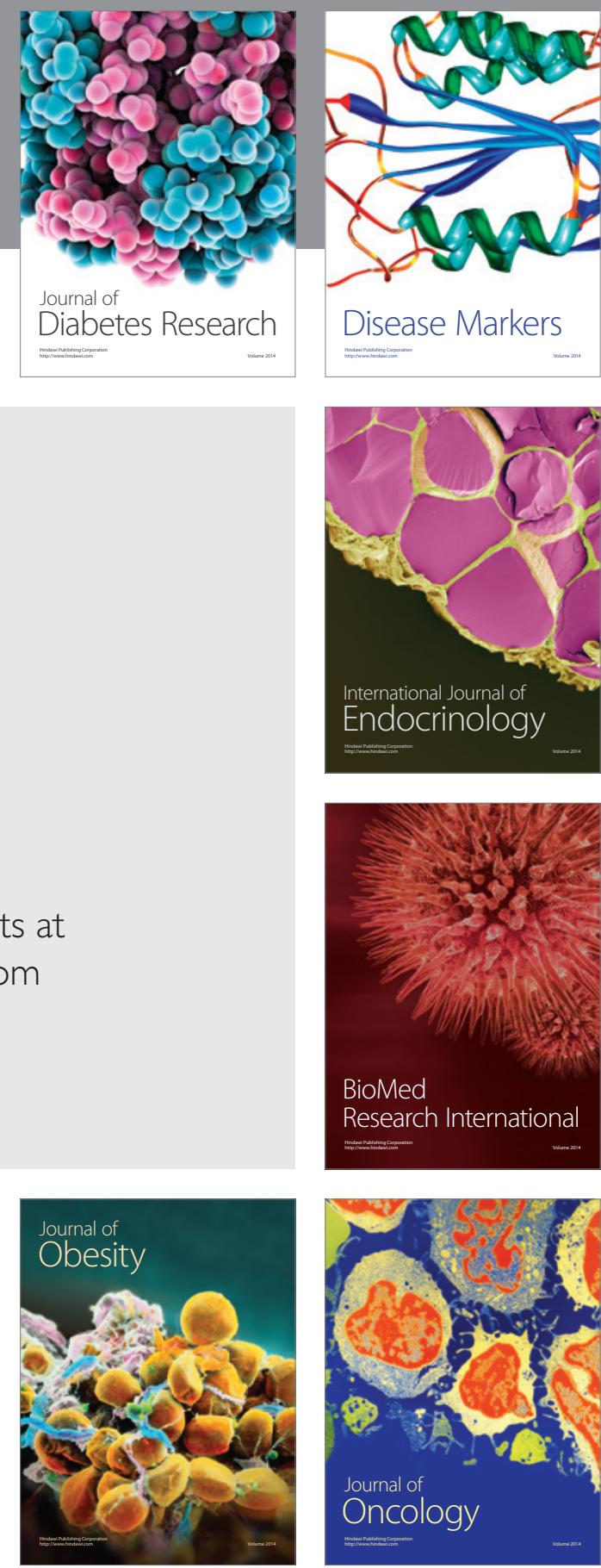

Disease Markers
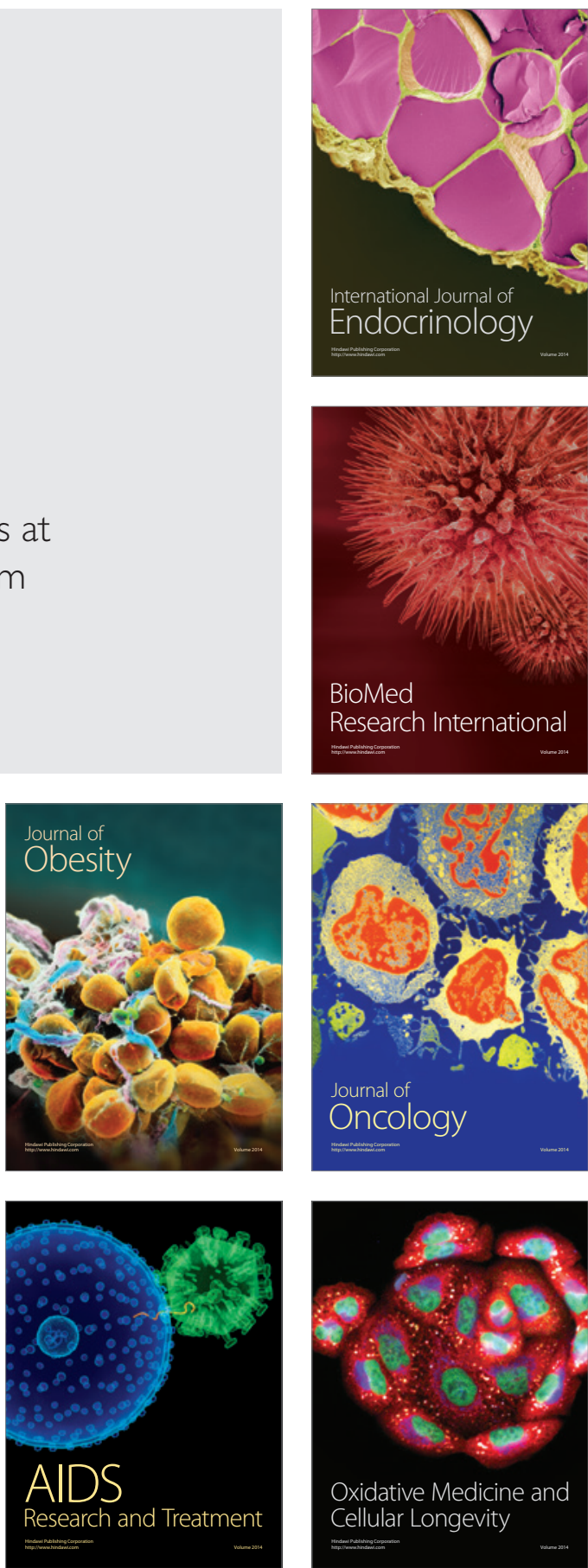